\title{
ULTRACAM observations of SDS 0926+3624: the first known eclipsing AM CVn star
}

\section{C.M. Copperwheat*}

Department of Physics, University of Warwick, Coventry, CV4 7AL, UK

E-mail: c.copperwheat@warwick.ac.uk

T.R. Marsh ${ }^{1}$, S.P. Littlefair ${ }^{2}$, V.S. Dhillon ${ }^{2}$, G. Ramsay ${ }^{3}$, A.J. Drake ${ }^{4}$, B.T. Gänsicke ${ }^{1}$, P.J. Groot ${ }^{5}$, P. Hakala ${ }^{6}$, D. Koester ${ }^{7}$, G. Nelemans ${ }^{5}$, G. Roelofs ${ }^{8}$, J. Southworth ${ }^{9}$, D. Steeghs ${ }^{1}$ and S. Tulloch ${ }^{2}$

${ }^{1}$ Department of Physics, University of Warwick, Coventry, CV4 7AL, UK

${ }^{2}$ Department of Physics and Astronomy, University of Sheffield, S3 7RH, UK

${ }^{3}$ Armagh Observatory, College Hill, Armagh, BT61 9DG, UK

${ }^{4}$ California Institute of Technology, 1200 E. California Blvd., CA 91225, USA

5 Department of Astrophysics, IMAPP, Radboud University Nijmegen, PO Box 9010, NL-6500

GL Nijmegen, the Netherlands

${ }^{6}$ Finnish Centre for Astronomy with ESO, Tuorla Observatory, Väisäläntie 20, FIN-21500

Piikkiö, University of Turku, Finland

${ }^{7}$ Institut für Theoretische Physik und Astrophysik, Universität Kiel, 24098 Kiel, Germany

${ }^{8}$ Harvard-Smithsonian Center for Astrophysics, 60 Garden Street, Cambridge, MA 02138, USA

${ }^{9}$ Astrophysics Group, Keele University, Newcastle-under-Lyme, ST5 5BG, UK

The AM Canum Venaticorum (AM CVn) stars are ultracompact binaries with the lowest periods of any binary subclass, and consist of a white dwarf accreting material from a donor star that is itself fully or partially degenerate. These objects offer new insight into the formation and evolution of binary systems, and are predicted to be among the strongest gravitational wave sources in the sky. To date, the only known eclipsing source of this type is the 28 min binary SDSS 0926+3624. We discuss here multiband, high time resolution light curves of this system, collected with ULTRACAM in 2006 and 2009. From light curve models we make the most precise parameter determinations for any AM CVn and determine the degree of degeneracy of the donor star; a key parameter in differentiating between the proposed formation paths for these objects.

High Time Resolution Astrophysics IV - The Era of Extremely Large Telescopes - HTRA-IV,

May 5-7, 2010

Agios Nikolaos, Crete, Greece

\footnotetext{
* Speaker.
} 


\section{Introduction}

The AM Canum Venaticorum (AM CVn) stars are ultra-compact binaries characterised by orbital periods of tens of minutes or less and optical spectra dominated by helium. These systems consist of a white dwarf accreting matter via a helium accretion disc from a significantly less massive and hydrogen defficient donor star, and in order to fit within the Roche lobe it is necessary for this donor to also be at least partially degenerate.

AM CVn stars offer new insights into the formation and evolution of binary star systems $[12,14]$. A key parameter is the donor mass, with the current paradigms for the binary formation path all imply partial degeneracy, to different degrees $[12,7,16]$. Close double-degenerate binaries are also one of the proposed progenitor populations of Type Ia supernovae [22, 23, 6]. Finally, the mass transfer in these systems is thought to be driven by angular momentum loss as a result of gravitational radiation. Due to their very short periods they are predicted to be among the strongest gravitational wave sources in the sky [13], and are the only class of binary with examples already known which will be detectable by the gravitational wave observatory LISA [21, 18].

Only $\sim 25$ objects of this class are currently known, and of these only SDSS 0926+3624 ([1], SDSS 0926 hereafter) has been observed to eclipse. SDSS 0926 has a period of $28 \mathrm{~min}$, with eclipses lasting $\sim 1 \mathrm{~min}$. The mean $g$-band magnitude of this system is $\sim 19.3$ [1], but there is considerable out-of-eclipse variation, characteristic of the superhumping behaviour seen in many AM CVns and CVs which is attributed to the precession of an elliptical accretion disc [24, 11, 19]. In 2006 and 2009 we took high time resolution observations of SDSS 0926 with the fast CCD camera ULTRACAM. The aim of these observations was to determine precise system parameters for this system, using techniques we have in the past successfully applied to normal CVs (e.g., $[10,3])$.

\section{Observations}

We observed SDSS 0926 with the high speed CCD camera ULTRACAM [5] mounted on the 4.2m William Herschel Telescope (WHT) in 2006 and 2009. ULTRACAM is a triple beam camera and all observations were made using the SDSS $u^{\prime}, g^{\prime}$ and $r^{\prime}$ filters. Average exposure times were $\sim 3 \mathrm{~s}$ in 2006 and 1.8s in 2009. The dead time between exposures for ULTRACAM is $\sim 25 \mathrm{~ms}$. The CCD was windowed in order to achieve this exposure time. A $2 \times 2$ binning was used in most of the 2006 data to compensate for conditions. The 2006 observations were taken on the nights of 1 - 3 March, with a small additional section of data obtained on 5 March. Weather conditions were reasonable, with seeing $\sim 1$ " and good transparency. The 2009 observations were taken on the nights of $1-3$ January, and conditions for these winter observations were on the whole poorer, with variable seeing and transparency. Due to conditions, only a small number of orbital cycles were observed on the first and third 2009 nights.

All of these data were reduced with aperture photometry using the ULTRACAM pipeline software, with debiassing, flatfielding and sky background subtraction performed in the standard way. The source flux was determined using a variable aperture (whereby the radius of the aperture is scaled according to the FWHM). Variations in transparency were accounted for by dividing the source light curve by the light curve of a nearby comparision star. The stability of this compari- 


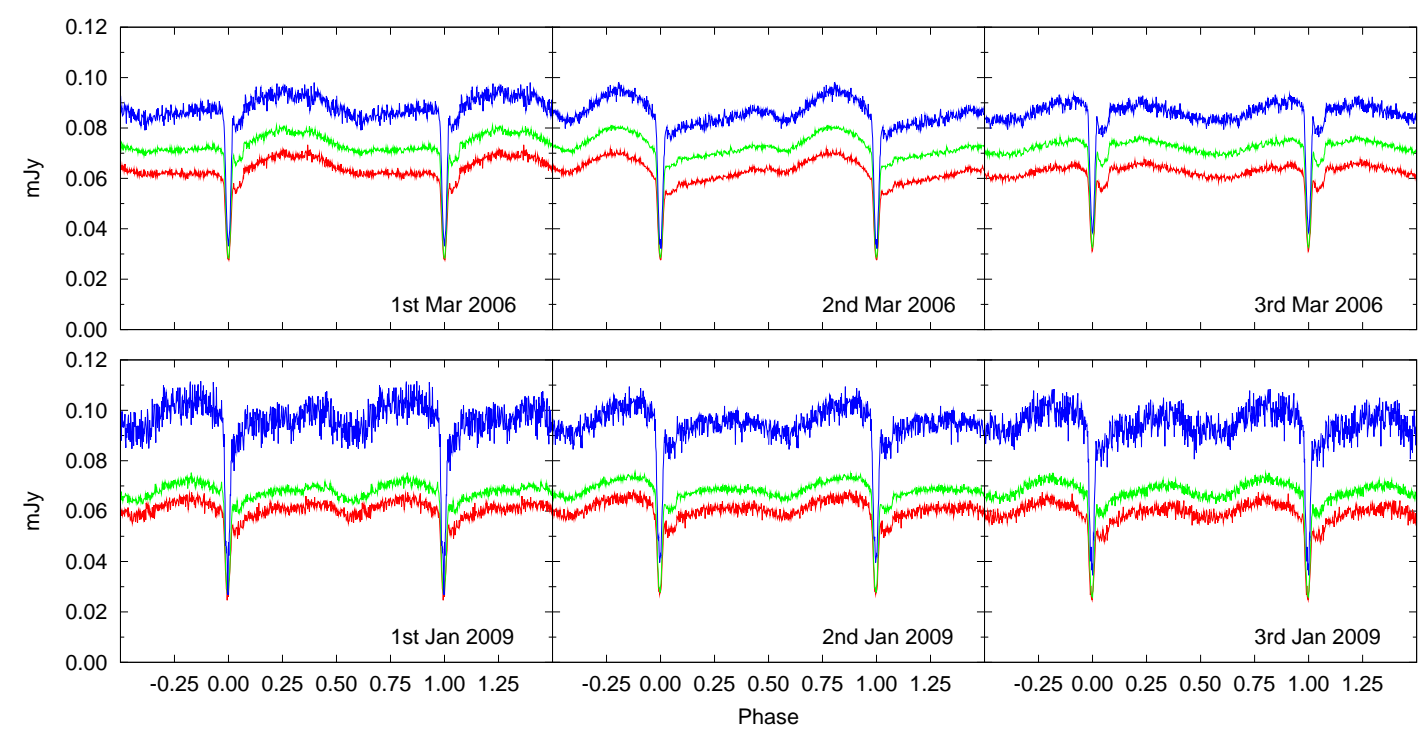

Figure 1: Phase folded and binned light curves, showing the superhump variation from night-to-night. In the top row we plot the first three nights of data collected in 2006. In the bottom row we plot the three nights of data taken in 2009. We plot separately the data in the $u^{\prime}$ - (top, blue), $g^{\prime}$ - (middle, green) and $r^{\prime}$-bands (bottom, red).

son star was checked against other stars in the field, and no variations were seen. We determined atmospheric absorption coefficients in the $u^{\prime}, g^{\prime}$ and $r^{\prime}$ bands and subsequently determined the absolute flux of our targets using observations of standard stars (from [20]) taken in evening twilight. We used this calibration for our determination of the apparent magnitudes of the source, although we present all light curves in flux units determined using the conversion given in [20]. Using our absorption coefficients, we extrapolate all fluxes to an airmass of 0 .

\section{Results}

We phase-folded the data on a night-by-night basis and plot the results in Figure 1. We omit from this plot the short section of data collected on 5th March 2006. It is evident in this plot that there are gross differences in the light curve from night to night over the course of the 2006 observations, which are due to the superhump precessing through the light curve resulting in the peak of the superhump emission being at different phases on different nights. If we examine the eclipse feature itself, we see that the primary eclipse is immediately followed by a distinct second, smaller eclipse (this is most apparent in the 3 March data). These two eclipses are of the white dwarf and the bright spot, respectively. The eclipses are preceeded by a small orbital 'hump' caused by the bright spot moving into the field of view. This is not immediately apparent since the bright spot is relatively weak in these data, so the out-of-eclipse variation is dominated by the superhump. In order to determine the parameters of the 2006 superhump, we fitted a model to the combined dataset with the eclipse features masked. The model consists of a combination of six 4-parameter sine functions: three for the superhump, fitting the primary frequency and the second and third harmonics, and three for harmonics of the orbital period, so as to fit any residual signal left after the masking of the eclipse features. 


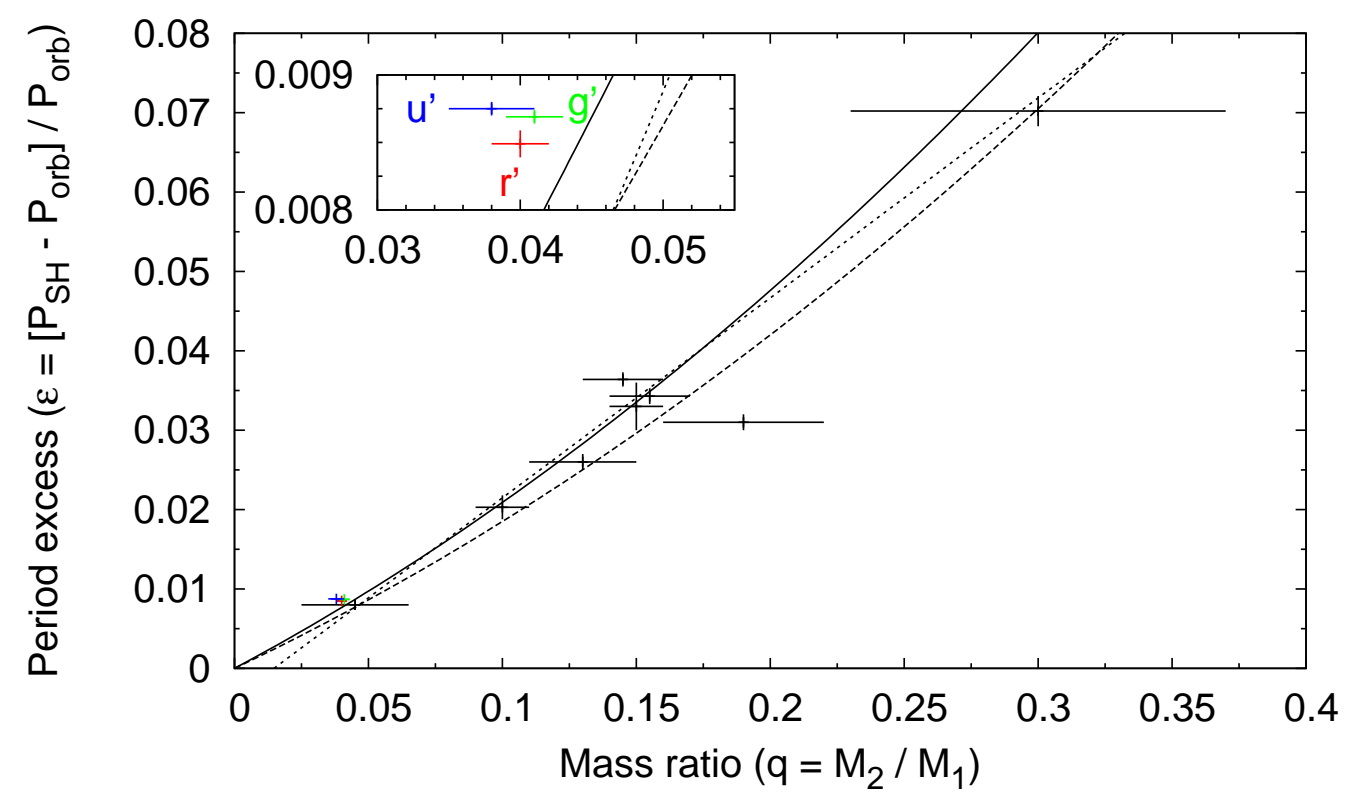

Figure 2: Mass ratio $q$ versus the superhump period excess $\varepsilon$. The blue, green and red points show our $u^{\prime}$-, $g^{\prime}$ - and $r^{\prime}$-band determinations, respectively. The region around these points is magnified in the inset. The solid line is the $\varepsilon=0.18 q+0.29 q^{2}$ relationship proposed by [15]. The dashed and dotted lines are the slightly modified relations propsed by [8] and [9] respectively. The black points are the eclipsing CVs listed as calibration sources in Table 7 of [15].

In contrast to 2006, in the 2009 data the shape of the out-of-eclipse light curve is roughly constant from night to night: we do not see the large variations caused by a superhump component precessing through the light curve. The shape of the light curve on all three nights is most similar to the 2 March 2009 data, with the peak of the emission shortly before the eclipse. Since the position of this peak does not vary from night-to-night it is most likely due to the bright spot, and thus there seems to be no significant superhump contribution in these data.

[15] suggested $\varepsilon=0.18 q+0.29 q^{2}$ as an empirical relationship between the superhump period excess $\varepsilon=\left(P_{S H}-P_{O r b}\right) / P_{O r b}$, and the mass ratio $q$. This relationship was calibrated using measurements of a series of eclipsing systems, listed in Table 7 of [15]. The calibration is potentially poor at low mass ratios (and underlying assumption of the relation is that $\varepsilon=0$ when $q=0$, but this is not determined empirically), and so SDSS 0926 is a strong test of this relationship. In Figure 2 we reproduce Figure 1 from [15], adding our measurements from the 2006 data in $u^{\prime}, g^{\prime}$ and $r^{\prime}$. We plot also the slightly modified relations of [8] and [9]. Our measurements of the period excess in SDSS 0926 are consistent with all of these relations to within their uncertainties.

\section{Light curve analysis}

In order to make precise parameter determinations we chose to combine our data into phasefolded and binned light curves. We began by preparing the 2006 data for fitting by subtracting the superhump modulation from the data, using the model fit described in Section 3. Since the superhump is not seen in 2009, this step is not necessary for this second epoch of data. Once the 

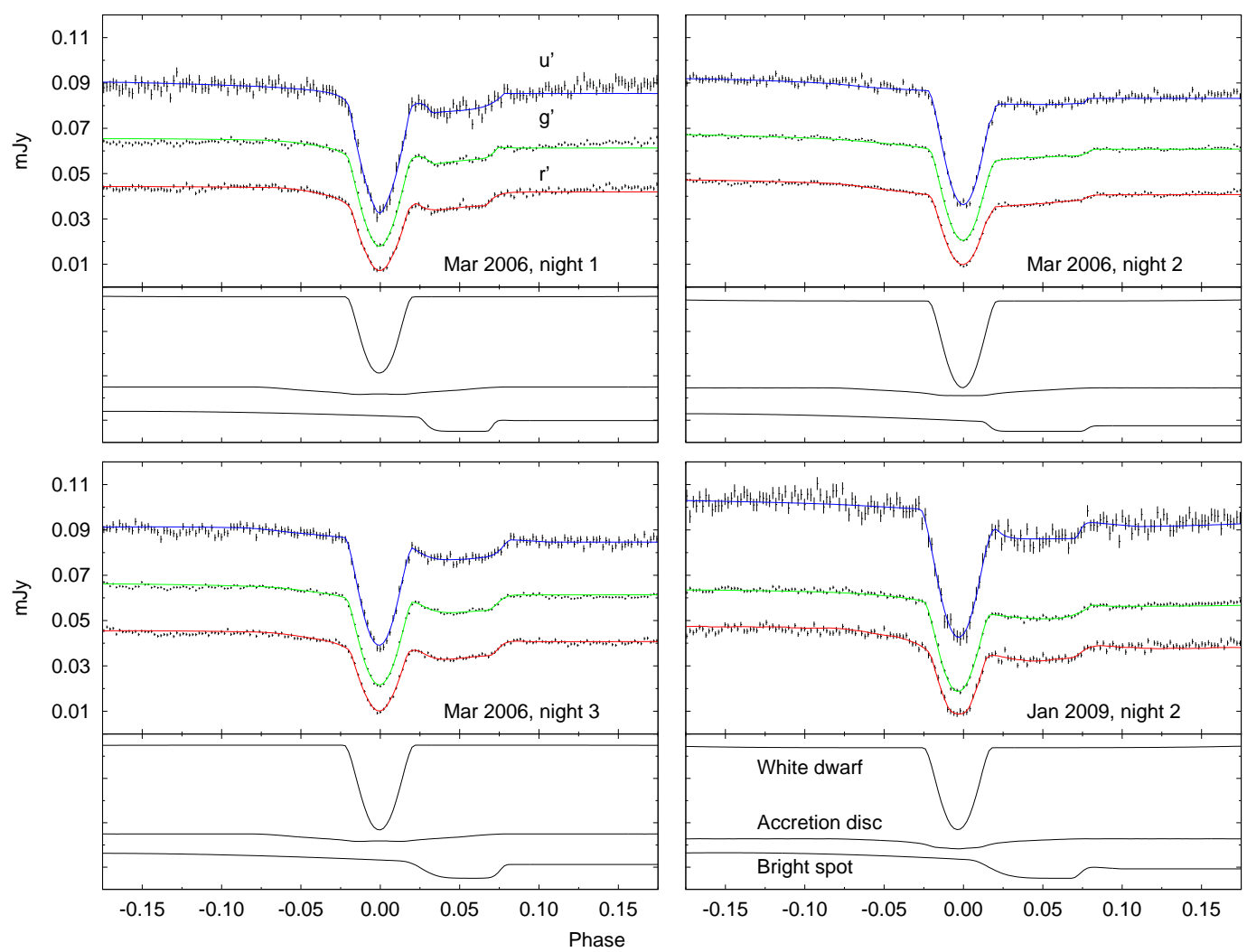

Figure 3: Phase-folded and binned light curves for the first, second and third nights of the 2006 observations, and the second night of the 2009 observations. For each we plot the three bands separately (top, $u^{\prime}$; middle, $g^{\prime}$; bottom, $r^{\prime}$ ). We plot the average flux in mJy against the binary phase, where a phase of 0 corresponds to the mid-eclipse of the white dwarf. We plot the datapoints with uncertainties in black, and the best model fits to these data in blue, green and red (for $u^{\prime}, g^{\prime}$ and $r^{\prime}$, respectively). For clarity we apply offsets of $0.005 \mathrm{mJy}$ to the $g$-band data and $0.01 \mathrm{mJy}$ to the $u$-band. For each night we also show underneath the light curve the three components of the $g^{\prime}$-band model plotted separately, showing the relative strengths of the bright spot, accretion disc and white dwarf. These three lines have also been offset for clarity.

superhump was subtracted it became clear that other binary parameters varied over the course of our observations, in particular the disc radius which changes significantly from night to night. We chose therefore to fit each night of data separately, rather than combine the entire WHT/ULTRACAM dataset. We omit the nights for which we only have small sections of data. The phase-folded and superhump-subtracted light curves and model fits are plotted in Figure 3.

We modelled the light curve with LCURVE, a code developed to fit light curves characteristic of eclipsing dwarf novae and detached white dwarf / $M$ dwarf binary stars [3]. In this code the binary is defined by four components: a white dwarf primary, a Roche-lobe filling secondary star, accretion disc and bright-spot. We first obtained an initial fit to each light curve using the simplex and Levenberg-Marquardt methods [17]. We then used a Markov Chain Monte Carlo (MCMC) algorithm for minimisation and determination of uncertainties [3]. A more complete description of these model fits is given in [4]. 


\section{Discussion}

If we assume a Roche-lobe filling donor star, the phase width of the white dwarf eclipse is then an observable quantity that is intrinsically linked to two physical properties: the mass ratio and the binary inclination. For a higher binary inclination the duration of the eclipse will be greater, thus to maintain the same phase width, as the inclination is increased, the size of the donor, and hence the mass ratio, must be decreased. There is therefore a unique relationship between these two properties [2]. This degeneracy can be broken since we have an additional geometric constraint due to the ingress and egress of the bright spot. The path of the accretion stream and hence the position of the bright spot is modified by the mass ratio. With this additional information we can determine both the mass ratio and inclination in this system.

The full results of our model fits are presented in [4]. We discuss here the $g^{\prime}$-band results, since these data have the highest signal-to-noise. Our findings in the other two bands are consistent with the values we report here. As discussed in Section 4 we fitted each night of data separately, and calculated the mass ratio $q=M_{2} / M_{1}$, inclination $i$ and white dwarf radius scaled by the binary separation $R_{1} / a$ for each night. We combined these individual determinations by calculating the weighted mean of each parameter, and find the mean mass ratio to be $q=0.041 \pm 0.002$, the inclination to be $i=82.6 \pm 0.3 \mathrm{deg}$ and $R_{1} / a$ to be $0.033 \pm 0.002$.

We used these determinations to calculate the remaining binary parameters. One additional piece of information that was needed for this is a mass/radius relation for the primary white dwarf. We determined this using an estimate of the white dwarf temperature which we made from our model fits, additional ultraviolet observations obtained with XMM-Newton and theoretical white dwarf cooling models. A complete description of this process is given in [4]. We subsequently found the binary separation to be $0.295 \pm 0.005$, the white dwarf and donor masses to be $0.85 \pm$ $0.04 M_{\odot}$ and $0.035 \pm 0.003 M_{\odot}$ respectively, and the donor radius to be $0.047 \pm 0.001 R_{\odot}$.

A donor mass of $0.035 \pm 0.003 M_{\odot}$ implies the donor is only partially degenerate: a fully degenerate donor in a system with this period would have a mass of $\sim 0.020 M_{\odot}$. There are three proposed formation paths for AM CVn binaries (white dwarf mergers, [12]; ex-helium stars, [7]; CVs with evolved donors, [16]) and they all imply partial degeneracy to different degrees. However, for an AM CVn with a period of 28 min the mass predictions are close in value, so our current findings do not strongly preclude any of the three formation channels. More precise determinations may be possible if we are able to make more observations of SDSS 0926 in its non-superhumping state, since even after subtracting the superhump from our data there is likely to still be some residual systematic effect. The discovery of new eclipsing AM CVn systems is also key, particularly at the short end of the period distribution where there is the biggest discrepancy between the various donor mass predictions.

\section{References}

[1] Anderson S. F., Haggard D., Homer L., Joshi N. R., Margon B., Silvestri N. M., Szkody P., Wolfe M. A., Agol E., Becker A. C., Henden A., Hall P. B., Knapp G. R., Richmond M. W., Schneider D. P., Stinson G., Barentine J. C., Brewington H. J., Brinkmann J., Harvanek M., Kleinman S. J., Krzesinski J., Long D., Neilsen Jr. E. H., Nitta A. Snedden S. A., Ultracompact AM Canum Venaticorum 
Binaries from the Sloan Digital Sky Survey: Three Candidates Plus the First Confirmed Eclipsing System, AJ, 2005, 130, 2230

[2] Bailey J., The dwarf nova Z Chamaeleontis. I - Photometry, MNRAS, 1979, 187, 645

[3] Copperwheat C. M., Marsh T. R., Dhillon V. S., Littlefair S. P., Hickman R., Gänsicke B. T., Southworth J., Physical properties of IP Pegasi: an eclipsing dwarf nova with an unusually cool white dwarf, MNRAS, 2010, 402, 1824

[4] Copperwheat C. M., Marsh T. R., Littlefair S. P., Dhillon V. S., Ramsay G., Drake A. J., Gänsicke B. T., Groot P. J., Hakala P., Koester D., Nelemans G., Roelofs G., Southworth J., Steeghs D., Tulloch S., SDSS J0926+3624: The shortest period eclipsing binary star, MNRAS, 2010 (submitted)

[5] Dhillon V. S.. Marsh T. R., Stevenson M. J., Atkinson D. C., Kerry P., Peacocke P. T., Vick A. J. A., Beard S. M., Ives D. J., Lunney D. W., McLay S. A., Tierney C. J., Kelly J., Littlefair S. P., Nicholson R., Pashley R., Harlaftis E. T., O'Brien K., ULTRACAM: an ultrafast, triple-beam CCD camera for high-speed astrophysics, MNRAS, 2007, 378, 825

[6] Iben I. J. and Tutukov A. V., Supernovae of type I as end products of the evolution of binaries with components of moderate initial mass (M not greater than about 9 solar masses) ApJS, 1984, 54, 335

[7] Iben I. J. and Tutukov A .V., Helium star cataclysmics, ApJ, 1991, 370, 615

[8] Kato T., Imada A., Uemura M., Nogami D., Maehara H. et al., Survey of Period Variations of Superhumps in SU UMa-Type Dwarf Novae, PASJ, 2009, 61, 395

[9] Knigge C., The donor stars of cataclysmic variables, MNRAS, 2006, 373, 484

[10] Littlefair S. P., Dhillon V. S., Marsh T. R., Gänsicke B. T., Southworth J., Baraffe I., Watson C. A., Copperwheat C., On the evolutionary status of short-period cataclysmic variables, MNRAS, 2008, 388,1582

[11] Luibow S.H., Simulations of tidally driven eccentric instabilities with application to superhumps, ApJ, 1991, 381, 268

[12] Nelemans G., Portegies Zwart S. F., Verbunt F., Yungelson L. R., Population synthesis for double white dwarfs. II. Semi-detached systems: AM CVn stars, AAP, 2001, 368, 939

[13] Nelemans G., Yungelson L. R., Portegies Zwart S. F., Short-period AM CVn systems as optical, X-ray and gravitational-wave sources, MNRAS, 2004, 349, 181

[14] Nelemans G., Yungelson L. R., van der Sluys M. V., Tout C. A., The chemical composition of donors in AM CVn stars and ultracompact X-ray binaries: observational tests of their formation, MNRAS, 2010, 401, 1347

[15] Patterson J., Kemp J., Harvey D. A., Fried R. E., Rea R., Monard B., Cook L. M., Skillman D. R., Vanmunster T., Bolt G., Armstrong E., McCormick J., Krajci T., Jensen L., Gunn J., Butterworth N., Foote J., Bos M., Masi G., Warhurst P., Superhumps in Cataclysmic Binaries. XXV. $q_{c r i t}, \varepsilon(q)$, and Mass-Radius, PASP, 2005, 117, 1204

[16] Podsiadlowski P., Han Z., Rappaport S., Cataclysmic variables with evolved secondaries and the progenitors of AM CVn stars, MNRAS, 2003, 340, 1214

[17] Press W. H., Numerical recipes in C++ : the art of scientific computing, Cambridge University Press, Cambridge, 2002

[18] Roelofs G. H. A., Nelemans G., Groot P. J., The population of AM CVn stars from the Sloan Digital Sky Survey, MNRAS, 2007, 382, 685 
[19] Simpson J. C. and Wood M. A., Time Series Energy Production in Smoothed Particle Hydrodynamics Accretion Disks: Superhumps in the AM Canum Venaticorum Stars, ApJ, 1998, 506, 360

[20] Smith J. A., Tucker D. L., Kent S., Richmond M. W., Fukugita M., Ichikawa T., Ichikawa S.-i., Jorgensen A. M., Uomoto A., Gunn J. E., Hamabe M., Watanabe M., Tolea A., Henden A., Annis J., The u'g'r'i'z' Standard-Star System, AJ, 2002, 123, 2121

[21] Stroeer A. and Vecchio A., The LISA verification binaries, Classical and Quantum Gravity, 2006, 23, 809 [astro-ph/0605227]

[22] Tutukov A. V. and Yungelson L. R., Evolutionary Scenario for Close Binary Systems of Low and Moderate Masses, NI, 1981, 49, 3

[23] Webbink R. F., Double white dwarfs as progenitors of R Coronae Borealis stars and Type I supernovae, ApJ, 1984, 277, 355

[24] Whitehurst R., Numerical simulations of accretion disks. I - Superhumps - A tidal phenomenon of accretion disks, MNRAS, 1988, 232, 35 